\title{
PLANEJAMENTO PARTICIPATIVO NO SUBPROGRAMA QEQ DO PADCT
}

Eucler B. Paniago

Instituto de Ciências Exatas e Biológicas, Universidade Federal de Ouro Preto, 35400-000 Ouro Preto - MG, Brasil

\begin{abstract}
JOINT STRATEGIC PLANNING IN THE CHEMISTRY AND CHEMICAL ENGINEERING SUBPROGRAM OF PADCT. The development of the Brazilian chemistry sector, during the last 30 years, is nowadays being attributed to PADCT (Science and Technology Development Program). Since the seventies, the Government took notice of the importance of research in chemistry for the Brazilian economy, therefore creating PADCT to support chemistry and chemical engineering among other areas of science and technology. Planning and implementation of the second phase of this program represented a real joint strategic planning. Since then, academic research and human resources education have experienced significant improvements. However, in the chemical trade, the deficit continues to grow, in spite of an almost constant ratio between importation costs and export revenues. Continued investments for research in the area remain necessary.
\end{abstract}

Keywords: PADCT; chemistry; planning.

\section{INTRODUÇÃO}

O atual estágio de desenvolvimento da Química no Brasil tem sido atribuído ao PADCT (Programa de Apoio ao Desenvolvimento Científico e Tecnológico ${ }^{1-4}$. Em 2001, o então Coordenador, na CAPES, da área de Química registrou em Editorial da Revista Química Nova2: "A maior contribuição para os notáveis progressos alcançados pela Química brasileira, no âmbito acadêmico, deve ser atribuída ao PADCT não somente em função dos investimentos, que possibilitaram, entre outras coisas, aquisições de equipamentos modernos e de grande porte, mas também pela sistemática de competição e avaliação e por beneficiar, além de grupos de pesquisa já consolidados, grupos em consolidação, programas de pós-graduação e, até mesmo, cursos de graduação. Não seria exagero dizer que a História da Química no Brasil pode ser dividida em duas fases, uma antes e outra depois do PADCT".

Cada Subprograma do PADCT, em cada uma de suas três etapas, possuía seu Grupo Técnico, composto de membros da comunidade acadêmica e/ou empresarial, cujas atribuições tinham início com a realização de um diagnóstico da situação da área, seguida da elaboração de um documento detalhando os resultados deste diagnóstico e as propostas para superação dos problemas encontrados. Implementava em seguida o programa definido, elaborando Editais e acompanhando a análise das propostas pelos Comitês Assessores organizados pelas Agências CAPES, FINEP e CNPQ, com nomes sugeridos pelas sociedades representativas da respectiva comunidade.

Participei deste Programa durante um longo período, primeiro como membro do Grupo Técnico do Subprograma de Química e Engenharia Química, GT/QEQ, desde seu planejamento inicial e durante a chamada "fase teste". Depois como seu Secretário Técnico durante sua implementação na fase-I, do planejamento da fase-II e finalmente como seu Coordenador na fase-II. Participei ainda, como representante do GT/QEQ, do Grupo Técnico de Informação em Ciência e Tecnologia. Com os arquivos desta época, pude elaborar este documento.

Apesar dos prejuízos que este trabalho provocou na minha carreira acadêmica na época, sinto-me recompensado pelos resultados deste trabalho e também pela medalha Simão Simão Mathias, recebida da diretoria da SBQ, em 1999.

"Professor aposentado dos Departamentos de Química da UFMG e UFOP e-mail: eucler@iceb.ufop.br
Atualmente, muitos dos profissionais da área, especialmente os mais jovens, desconhecem a situação em que a Química se encontrava anteriormente a esse Programa. Entre as várias contribuições feitas pelo PADCT à Química relacionadas pelo Coordenador da CAPES, faltaram, por exemplo, o apoio dado às Bibliotecas de Química bem como o planejamento e a implementação do Subprograma QEQ, realizado em estreito contato com a comunidade.

\section{HISTÓRICO}

Desde a década de 70, do século passado, o desequilíbrio na balança comercial de produtos químicos mostrou ao governo brasileiro que a pesquisa química brasileira é muito pequena perante as necessidades de um parque industrial moderno. Visando expandir e diversificar as atividades de pesquisa, iniciativas de cooperação internacional, como o Programa Multinacional de Química, apoiado pela Organização dos Estados Americanos (OEA) e o Programa de Cooperação entre a National Academy of Sciences (NAS) e o CNPq (1969/1975) foram implementadas. Este último, embora restrito aos estados do Rio de Janeiro e São Paulo, produziu importantes resultados científicos especialmente neste último estado. Em "Química Inorgânica, o impacto desses programas foi marcante, com envolvimento de pesquisadores como Larry Thompson (1969), John Malin (1969-72) e Henry Taube (1969-75) em São Paulo, e Harry Gray (1969-72) no Rio de Janeiro, e o início de pesquisas na área de reatividade química, utilizando técnicas de cinética rápida e espectroscopia. Sob a supervisão de Henry Taube, foi implantado o laboratório de cinética e mecanismos de reações inorgânicas no Instituto de Química da USP. Nesse laboratório foram iniciadas novas linhas de pesquisa focalizando a química de aminas de rutênio e ósmio, ainda bastante incipiente, as quais viriam a se expandir para a química dos cianoferratos, completando a tríade dos elementos. A influência de Taube na Química Inorgânica brasileira não ficou limitada à sua atuação pessoal, tendo sido principalmente exercida através de interações com pesquisadores formados em sua Escola, em âmbito nacional e internacional, a partir dos anos 70. Esse fato explica a elevada densidade de trabalhos sobre a química do rutênio e dos cianoferratos em nosso país, até os tempos atuais"5

Nos anos 80, criou-se o PRONAQ (1980-1985), um programa de âmbito nacional que visava apoiar a infra-estrutura de centros de pesquisa e promover a criação e implantação de novas linhas de pesquisa relacionadas com a atividade química industrial ${ }^{6}$. 
A propósito da criação deste programa é interessante recordar o que disse numa conferencia em Recife o bioquímico, ex-reitor da UFPE, Marcionilo de Barros Lins, referindo-se à sua universidade: "se crescemos em física, se crescemos em matemática, ao ponto de nos tornarmos centro de excelência, por que não conseguimos a mesma performance em química? O que nos consola é que isso não é uma questão de Pernambuco. No Brasil todos nós temos, realmente, um brutal déficit de químicos, em matéria de competência química, para desenvolvimento de tecnologia. Daí, as providências de um investimento maciço na área da química, tendo sido criado, inclusive, pelo Ministro Delfim Neto, o PRONAQ. Foi o próprio Ministro quem assinou o programa oficial de lançamento do PRONAQ, no CNPq em Brasília"7.

Este programa resultou de uma proposta inicial, felizmente abortada, de criação pelo CNPq de um Instituto Nacional de Pesquisas Químicas. Seu maior sucesso talvez tenha sido constituir-se no embrião da primeira etapa do Subprograma de Química e Engenharia Química do PADCT, iniciado em 1985.

Também em 09/1985, a Comissão para Planejamento do Governo (COPAG), criada pelo Governo que se instalava, deixou claro que o "estabelecimento de uma estratégia de ciência e tecnologia para a química depende em grande parte da política de desenvolvimento econômico a ser adotada para o setor industrial correspondente", sendo "impensável o desenvolvimento de vários outros setores da economia, como a microeletrônica, metalurgia, agropecuária, etc, sem uma capacitação tecnológica sólida em Química".

A COPAG descreveu ainda, resumidamente, o quadro químico brasileiro: "O que se observa no Brasil em termos de Ciência e Tecnologia, em todo o campo da Química, é o desempenho considerado deficiente, principalmente quando comparado ao de outras ciências exatas e disciplinas tecnológicas, notadamente a Física e alguns ramos da Engenharia. Os esforços do CNPq/FINEP, empreendidos nos últimos anos não conseguiram modificar o quadro geral".

Em 06/1986, a SBQ, atendendo solicitação do MCT, produziu um documento onde as metas a serem atingidas na área de Química no Brasil para o triênio 1987/1989 foram delineadas. Transcreve-se deste documento, o trecho que evidencia a ausência de uma massa crítica de pesquisadores condizente com as necessidades de desenvolvimento do país: "O número de doutores, hoje em atividade no país, está em torno de quinhentos e cinquenta na área de Química e de noventa na área de Engenharia Química. Se for feita uma projeção com base no ritmo atual de formação de pós-graduados nestas duas áreas e se os cursos de pós-graduação não tiveram uma injeção de recursos financeiros a fim de que recuperem sua capacidade de pesquisa, o país chegará ao século XXI com um contingente de mil e cem doutores na Química e de cento e cinquenta na Engenharia Química, números estes muitíssimo aquém das necessidades de desenvolvimento autóctone".

A premência da expansão do contingente de químicos e engenheiros químicos qualificados encontrou respaldo em 1986 nos diversos Comitês Assessores das áreas de Ciências Exatas do CNPq, conscientes da necessidade de um desenvolvimento científicotecnológico global e harmônico (PPAP, 1986).

Ainda em setembro/1986, o Comitê Assessor de Química do $\mathrm{CNPq}$ detalhou para a Presidência desse órgão as medidas que, a seu juízo, deveriam ser observadas pelo mesmo, a fim de mais rapidamente incrementar o número de pesquisadores competentes dedicados à Química, a saber:

estimular prioritariamente a formação de doutores no exterior; dar, simultaneamente, condições aos cursos de pós-graduação para a formação de mais doutores em Química no Brasil, invertendo-se a situação atual em que predomina a formação de mestres; estimular os estágios de pós-doutorado no exterior; estimular a vinda de professores visitantes do exterior para catalisar um maior desenvolvimento da pesquisa química no Brasil;

estimular o intercâmbio científico e docente entre as instituições brasileiras a fim de diminuir seu distanciamento e provincianismo;

favorecer a absorção de recém-doutores por Universidade diferente daquela em que se formou, para evitar o vício da endogenia dentro das universidades nacionais.

O Comitê ressaltou, ainda, ser "fundamental dar aos pesquisadores acadêmicos brasileiros as condições apropriadas e competitivas de trabalho, que implicariam em:

1. manter e renovar equipamentos (incluindo peças de reposição e outros);

2. adequar a infra-estrutura global das atividades de pesquisa (oficinas de vidro, eletrônica, mecânica, pessoal técnico qualificado e outros);

3. melhorar qualitativa e quantitativamente as bibliotecas de Química no país, que estavam, em sua maioria, em estado deplorável;

4. criar facilidades para a importação de reagentes, outros materiais de consumo, equipamentos e peças;

5. estimular os departamentos e/ou institutos de química a promover um planejamento global visando ao seu desenvolvimento orgânico em termos de crescimento e implantação das diversas áreas".

Essas medidas receberam, em setembro de 1986, o aval dos Presidentes de Associações e Sociedades de Química e Engenharia Química, em reunião conjunta no $\mathrm{CNPq}$, durante a qual tornaram essas medidas até mais específicas em alguns casos, como se observa a seguir:

estimular a formação de doutores na área da química sem a obrigatoriedade do mestrado;

atender a toda a demanda qualificada de bolsas dos cursos

de pós-graduação;

criar mecanismos de indução para formação de doutores no exterior em áreas da química carentes em recursos humanos; atuar junto ao MEC no sentido de reverter a atual carreira

do magistério superior tornando-a meritocrática;

criar seguro social para bolsistas de PG bem como contar tempo de serviço;

fixar o valor da bolsa de doutorado igual ao salário de Pro-

fessor Assistente I das Universidades autárquicas.

Muitas dessas medidas começaram a ser implementadas, tendo o PADCT sido o Programa que mais contribuiu para que algumas delas ocorressem.

\section{PADCT-I}

Na sua primeira fase, o Subprograma QEQ (iniciado em1984), deixou registrado no seu Documento Básico que os principais problemas que, em maior ou menor extensão, dificultavam a evolução do setor químico e o seu desenvolvimento tecnológico eram:

- Produção insuficiente de certas especialidades químicas.

- Ausência de suporte tecnológico endógeno adequado.

- Declínio nas atividades de pesquisas científicas nas Universidades e nos Institutos Tecnológicos Estatais.

- Atividade insuficiente de P\&D nas Empresas.

- Inadequação qualitativa e quantitativa dos recursos humanos envolvidos nas atividades de C\&T Química.

- Dificuldades de infra-estrutura de pesquisa (acesso e manutenção de equipamentos).

Estes problemas foram expostos e analisados detalhadamente, no que diz respeito ao seu alcance, suas causas e consequências. Como resultado o QEQ estabeleceu como objetivo geral "o fortale- 
cimento, em todos os seus segmentos e de forma harmoniosa, da base científica e tecnológica do setor químico brasileiro", desdobrando-o nos seguintes objetivos específicos:

1. Conhecimento Científico: Aumentar a produção de conhecimento científico químico básico e de tecnologia química voltada para as necessidades nacionais.

2. Recursos Humanos: Incrementar a melhoria de qualidade, diversificação e adequação às necessidades nacionais dos recursos humanos envolvidos em todos os segmentos de atividade de pesquisa do setor químico.

3. Pesquisa e Desenvolvimento: Estabelecer facilidades instrumentais, em técnicas estabelecidas e de ampla utilização (RMN, espectrometria de massas, difração de raios X, análise elementar, espectrometria infravermelho, Raman, fluorescência de Raios $\mathrm{X}$ ), necessárias para o trabalho de pesquisa e desenvolvimento em Química, melhorando assim a infra-estrutura laboratorial para P\&D em Química.

4. Subsídios: Apoiar atividades geradoras de subsídio à coordenação e planejamento do SNDCT, e seu gerenciamento.

5. Ações de Extensão Tecnológica: Apoiar ações de extensão tecnológica que visem a integração das atividades do setor produtivo e de P\&D. Essas ações seriam implementadas através de assessoramento técnico e tecnológico de instituições de P\&D capacitadas, dirigidas principalmente para a pequena e média empresa, visando a melhoria da qualidade do produto, adequação às atuais necessidades do país e inovação da tecnologia utilizada.

\section{PADCT-II}

A elaboração do Documento Básico do Subprograma QEQ na segunda fase do PADCT representou um exemplo de persistência do GT, que precisava conciliar os desejos da comunidade dos químicos com os interesses do governo brasileiro e as diretrizes do Banco Mundial, representado pelo seu assessor Aron Kuppermann, um paulista, formado em Engenharia Química e Civil pela USP, Professor de Físico-Química no Instituto de Tecnologia da California. Este trabalho representou um verdadeiro e inédito exercício de planejamento participativo, cujo desenvolvimento merece ser detalhado.

O trabalho teve início em abril de 1988, quando o GT iniciou uma ampla consulta à comunidade da área para avaliar os resultados obtidos pelo Subprograma na primeira fase do Programa (PADCT-I) e a pertinência de sua continuidade, bem como para a definição de prioridades.

Com estes objetivos, os membros do GT reuniram-se com pesquisadores, tecnólogos e empresários em pequenas reuniões, procurando colher o maior número de subsídios, reivindicações e sugestões. Elaborou-se um questionário, cujas respostas foram discutidas nas reuniões ou encaminhadas diretamente ao GT.

Uma primeira versão do Documento foi apresentada à comunidade, em 1988, durante as reuniões da SBPC (julho, em São Paulo), da ABEQ (julho, em São Paulo) e da ABQ (outubro, em Porto Alegre). Esta versão, após sofrer algumas modificações sugeridas pela comunidade, foi aprovada, em principio, pela chamada Comissão Transitória do PADCT em 15/09/1988.

Uma segunda versão teve sua elaboração concluída pelo GT em 15/05/1989, atendendo orientação da Secretaria Executiva no sentido de reduzir o orçamento inicialmente previsto. Esta segunda versão foi novamente apresentada à comunidade durante a reunião anual da SBPC, realizada em julho de 1989, em Fortaleza.

Uma terceira versão foi concluída pelo GT em 21/08/1989, atendendo orientação de consultores do Banco Mundial, no sentido de detalhar melhor o orçamento. Esta versão foi minuciosamente discutida com a Missão do Banco Mundial, durante reunião realizada em São Paulo nos dias 31/10 e 01/11/89. Como resultado dessa análise, o GT/QEQ reuniu-se novamente em Brasília, nos dias 04 e 05/12/89, para concluir a elaboração da versão final.

Participaram da elaboração deste Documento, desde o início, todos os membros do GT, bem como ex-coordenadores de Comitês Assessores e do GT, conforme a relação a seguir:

Representantes da comunidade: Eduardo Humeres (Química/ UFSC), Gilberto Fernandes de Sá (Química/UFPE), Jailson Bittencourt de Andrade (Química/UFBA), Jaime Rabi Nallar (NPPN/ UFRJ), José Carlos Gerez (Codetec/Campinas), José Osvaldo Beserra Carioca (Engenharia/UFCE), Olívia Ottoni Campos (Química/Unb), Pedro Wongtschowski (Ultra Química), Odette Vieira Gonçalves de Souza (Engenharia/UFMG-Coordenadora) e Eucler B. Paniago (Química/UFMG-Secretário Técnico)

Representantes das Agências: José Jorge Campello R. Pereira/ Eliane Louzão (Finep), Maria Aparecida Hugo Cagnin (CNPq), Massayoshi Yoshida (Capes) e Nícia Maria Mourão Henrique/ Newton Goulart (SDI/MIC)

Ex-Coordenadores (do Grupo Técnico e de Comitês Assessores): Fernando Galembeck (Química/Unicamp), Raimundo Bràz Filho (Química/UFRRJ), Leonardo Nogueira (CENPES/Petrobrás) e Luiz Antonio d'Avila (Grupo Química)

\section{SITUAÇÃO DA QUÍMICA EM 88/89}

Tendo analisado a situação em que então se encontrava a Química Brasileira (compreendendo Química e Engenharia Química), o GT registrou no Documento Básico:

- é pequeno o número de pesquisadores da área, mesmo quan-

do comparado com outras áreas do conhecimento

- $\quad$ seus objetivos são conservadores e pouco ambiciosos e, finalmente

- existe ainda um isolamento entre a área acadêmica e a industrial.

Registrou ainda: "esta situação é perversa, uma vez que tende a perpetuar dependências tecnológicas do setor químico. A situação de atraso em que se encontra a química brasileira é também fruto do modelo de desenvolvimento adotado e da falta de diálogo entre a comunidade acadêmica, os empresários e o governo. Neste contexto, cabem à comunidade acadêmica e empresarial dirigir seus esforços para identificar e delinear as ações básicas a fim de estabelecer e implementar o planejamento governamental neste setor da Ciência e Tecnologia".

Analisando críticas e sugestões recebidas da comunidade, observou: "o PADCT tem sido uma experiência valiosa, pois seus objetivos e sua sistemática operacional são fruto de ampla discussão nas comunidades acadêmica e empresarial, sendo sua transparência uma das razões centrais de seu sucesso. Está, por isso mesmo, sujeito a críticas e reflexões, que permitem seu permanente aprimoramento e ajuste".

Em relação à postura então predominante na comunidade acadêmica, alertou: "é uma tradição acadêmica que a universidade seja a instituição onde se cria e difunde o conhecimento e onde se formam recursos humanos. Em algumas áreas da química, esta difusão permanece intramuros ou com uma ótica de contribuição apenas ao conhecimento universal".

Justificando a postura que iria assumir, advertiu: "pelo seu caráter abrangente, o PADCT oferece uma oportunidade impar para que o País possa colocar em prática uma política de desenvolvimento científico e tecnológico para a área de Química e Engenharia Química. Por isso, uma nova postura deve ser praticada uma vez que é notório que a ciência somente teve impacto decisivo no desenvolvimento quando os conhecimentos básicos foram dinamicamente apropriados, 
resultando em produtos e serviços para satisfazer às necessidades crescentes da humanidade. A revolução propiciada pelas novas tecnologias está sendo um exemplo didático do processo de criação e apropriação do conhecimento. Hoje, mais do que nunca, o conhecimento básico está próximo de sua aplicação e os novos produtos estão tornando obsoletos outros que já duram dezenas de anos. A missão da inteligência nacional - comprometida com o País - é, portanto, buscar caminhos próprios que compreendem:

- considerar a natureza de um País tropical como fonte de inspiração para programas de ação que atendam às necessidades da sociedade brasileira.

- contribuir para e apropriar-se do conhecimento universal com uma visão crítica que não leve a dependências".

Registrou ainda: "esta é uma missão que não está sendo cumprida em toda a sua extensão e se constitui numa oportunidade de contribuir para o conhecimento universal e, ainda, a resolver problemas locais.Uma capacidade tecnológica sólida em química é essencial para o desenvolvimento de vários outros setores de grande importância sócio-econômica (medicina, biotecnologia, microeletrônica, agropecuária, novos materiais, etc) e para a preservação do meio ambiente. Como o número de doutores, hoje em atividade, é muito pequeno (da ordem de 500 doutores em Química e 100 em Engenharia Química), é imprescindível um aumento significativo destes pesquisadores através de programas integrados de formação de pessoal.

Certamente, é um grande desafio que a universidade, o meio industrial, o governo e a sociedade devem enfrentar juntos, com criatividade e tolerância, sob pena de o país continuar sujeito a uma dependência crescente, cada vez mais sofisticada, levando-o a ficar à margem do progresso universal".

De forma sucinta definiu-se que o objetivo geral do Subprograma QEQ seria então: "o desenvolvimento do setor químico brasileiro, através do fortalecimento integrado de sua base científica e tecnológica, com ênfase no treinamento de recursos humanos".

Justificando o objetivo definido, acrescentou-se: "esta integração permitirá a evolução tecnológica do conhecimento básico gerado no país, configurando oportunidades de surgimento de produtos a médio e longo prazos. Esta integração permitirá ainda:

1. a expansão das atividades de criação e de absorção, domínio, reprodução e melhoria das tecnologias em uso na indústria química brasileira;

2. desenvolvimento de tecnologias de processo necessárias ao desenvolvimento do país, com ênfase em tecnologias não disponíveis para compra no mercado internacional e em tecnologias que atualmente estão em fase inicial de desenvolvimento;

3. incremento do esforço nacional em desenvolvimento de tecnologia de aplicação e de produto, permitindo uma melhor adequação dos produtos às características do mercado brasileiro".

\section{OBJETIVOS ESPECÍFICOS DEFINIDOS}

\section{Primeiro objetivo}

Apoiar a formação de recursos humanos, através do exercício da pesquisa científica e de cursos formais, principalmente no nível de posgraduação, das universidades, centros de pesquisa e indústrias nacionais visando aumentar sua competência científica e tecnológica.

\section{Estratégia}

Expandir e acelerar a formação de mestres e doutores que possam dar o suporte necessário ao desenvolvimento da área, assim como formar técnicos e especialistas de apoio à pesquisa.

\section{Meios}

Esta estratégia contará com os seguintes meios:

1. melhorar a infra-estrutura dos cursos de pós-graduação;

2. melhorar a infra-estrutura dos cursos de graduação, instrumentalizando as universidades carentes para aprimorar seus aspectos didáticos e experimentais, incentivando ainda a realização de estágios de graduandos em indústrias e centros de pesquisa;

3. prover recursos para concessão de bolsas de Doutorado, Mestrado e Iniciação Científica, associadas a projetos, em número complementar às concedidas pelas Agências de fomento, de forma a garantir as metas fixadas. Aumentar o intercâmbio com o exterior através da vinda de pesquisadores visitantes e o intercâmbio de pesquisadores no País, criar áreas interdisciplinares, e fomentar a troca de experiências através de encontros, cursos, conferências, simpósios e seminários que contem com a participação de pesquisadores do país e do exterior;

4. aumentar os recursos para projetos de pesquisa comprometidos com a formação de recursos humanos, em especial em áreas de reconhecida carência ou prioritárias;

5. contribuir para a redução do tempo médio de titulação nos cursos de mestrado e doutorado, viabilizando a importação de insumos e a manutenção e aquisição de equipamentos em tempo hábil;

6. formar e aperfeiçoar o pessoal técnico de apoio à pesquisa.

\section{Metas}

1. Apoiar a infra-estrutura dos cursos de posgraduação.

2. Agregar, pelo menos, 75 novos doutores em química e engenharia química, formados no exterior, seja pelo apoio à sua formação ou pela contratação, no exterior, de elementos já titulados.

3. Incrementar a oferta de bolsas para formação, no país, de especialistas, mestres e doutores.

4. Apoiar a infra-estrutura de 40 cursos de graduação (20 de Química e 20 de Engenharia Química).

Além disto, a nova legislação do país, a qual obriga as empresas de maior porte a aplicarem $2 \%$ do seu faturamento em P\&D, ocasionará a contratação por estas de pessoal com nível de doutorado. Dessa maneira, embora seja desejável formar um número muito maior do doutores para a área, dadas as características de enfoque do PADCT e a quantia limitada de recursos, o número de 75 bolsas foi considerado apropriado pelo GT.

\section{Segundo objetivo}

Incentivar o Desenvolvimento do Conhecimento Científico Básico, especialmente nas Instituições geradoras de Recursos Humanos. O desenvolvimento de conhecimento científico básico deve ser fortemente incentivado, fortalecendo a formação de recursos humanos não só para permitir o desenvolvimento da ciência enquanto ciência, mas também para fundamentar os esforços de desenvolvimento tecnológico, visando o crescimento auto-sustentado do setor.

\section{Estratégia}

Aumentar a produção do conhecimento científico em química, procurando promover a interação da Química e da Engenharia Química com outras áreas do conhecimento.

\section{Meios}

Serão utilizados os seguintes meios:

apoiar grupos de pesquisa em Química e Engenharia Química com atividades de grande conteúdo científico e que abordem, com originalidade, problemas relevantes ao desenvolvimento científico 
e tecnológico do País.

Serão apoiadas dois tipos de propostas:

- as apresentadas por grupos consolidados de pesquisa, cuja produção nos últimos cinco anos, tenha mérito científico, regularidade e seja expressiva;

- $\quad$ as apresentadas por grupos em fase de consolidação, liderados por pesquisador qualificado.

Apoio plurianual (por até 5 anos), com avaliação periódica, no montante necessário para:

- atender às necessidades dos grupos consolidados;

- incentivar a consolidação de novos grupos.

Estes auxílios deverão atender a um amplo espectro de necessidades dos grupos beneficiados, inclusive equipamentos do porte necessário às suas atividades e bolsas de estudos.

- Estímulo à interdisciplinaridade através do apoio de programas onde o desenvolvimento do conhecimento básico resulte da interação de pesquisadores de várias áreas.

- Estímulo às empresas para investir em pesquisa básica.

- Estimulo à disseminação dos resultados de pesquisa, visando seu melhor aproveitamento.

\section{Metas}

- Apoiar, por até cinco anos, até 25 grupos de pesquisa diferentes, consolidados.

- Apoiar, por até cinco anos, até 40 novos grupos de pesquisa diferentes.

\section{Terceiro objetivo}

Integrar a Indústria com as Universidades e Centros de Pesquisa, através do incentivo ao desenvolvimento de trabalhos integrados e conjuntos entre universidades, centros de pesquisa e indústrias, dando a uns e outros os benefícios do diálogo e da apropriação dos conhecimentos reciprocamente disponíveis.

\section{Estratégia}

Estabelecer vínculos entre a Indústria, Universidades e Instituições de Pesquisa.

Este objetivo é orientado no sentido de estabelecer laços de interação entre interlocutores já capacitados para um entrosamento de alto nível. Nesta categoria de interlocutores incluem-se, de um lado, indústrias com centros de P\&D estabelecidos, empresas de alto conteúdo tecnológico com tradição em desenvolvimento e, de outro lado, laboratórios de pesquisa de Universidades ou Institutos de Pesquisa com pesquisadores - especialistas que tenham reconhecida competência.

Tendo em vista o caráter indutivo do PADCT, cumpre direcionar o campo de atuação no que se refere a projetos aplicados para aqueles correlacionados com o desenvolvimento da Química Fina, entendida como sendo a química de produtos industriais que possuem um alto valor agregado e um elevado conteúdo tecnológico.

\section{Meios}

O programa propiciará a utilização dos seguintes meios:

1. estimular o desenvolvimento de projetos de pesquisa de interesse de indústrias químicas, através da preparação de teses de posgraduação orientadas por professores universitários especialistas na área;

2. incentivar grupos de pesquisa de Universidades ou Institutos de Pesquisa interessados em continuar o desenvolvimento de conhecimentos básicos, que configurem oportunidades de desenvolvimento de novos produtos ou processos, a buscarem interações com empresas da área;
3. induzir atividades que favoreçam contatos frequentes dos profissionais das empresas com pesquisadores para troca de informações em áreas de interesse recíproco. Estes encontros deverão resultar na redefinição constante das novas fronteiras tecnológicas relevantes para o país;

4. promover o funcionamento de pequenas plantas-piloto para estudos de transposição de escala, especialmente em Centros de $\mathrm{P} \& \mathrm{D}$ onde estejam sendo desenvolvidas pesquisas inovadoras;

5. estimular estudos integrados de prospecção tecnológica para definição de novas estratégias.

A meta correspondente a este objetivo é a de apoiar até 35 projetos.

\section{Quarto objetivo}

Apoiar Programas Institucionais de Pesquisa e Desenvolvimento, canalizando recursos para a melhoria da infra-estrutura e para custeio de programas de $\mathrm{P} \& \mathrm{D}$ voltados para o desenvolvimento da tecnologia e das indústrias nacionais.

\section{Estratégia}

Contemplar projetos que apresentem a garantia de posterior transferência dos resultados ao setor industrial, visando o desenvolvimento tecnológico da indústria química.

Neste sentido serão utilizados os seguintes meios: suporte a laboratórios de $\mathrm{P} \& \mathrm{D}$, em instituições públicas ou privadas de capital nacional, priorizando aquelas com capacitação suficiente para atuar em todo o espectro de um desenvolvimento tecnológico (pesquisa aplicada, desenvolvimento piloto, anteprojecto de engenharia), mediante:

1. projetos de infra-estrutura laboratorial;

2. projetos de estabelecimento e fortalecimento de unidades de desenvolvimento experimental;

3. .projetos de fortalecimento de unidades capacitadas a trabalho analítico, verificação de conformidade e controle de qualidade não rotineiro;

4. apoio a projetos integrados de $\mathrm{P} \& \mathrm{D}$ (em instituições públicas ou privadas nacionais) para o desenvolvimento de setores específicos da indústria nacional, mediante estímulo à pesquisa integrada que associe instituições de P\&D e empresas nacionais;

5. suporte a projetos de desenvolvimento da indústria química nacional;

6. suporte a projetos de desenvolvimento em instituições de pesquisa tecnológica;

7. estímulo a capacitação da indústria de bens de capital para o desenvolvimento de equipamentos para a indústria química mediante:

8. projetos que contemplem associação entre empresa de bens de capital e instituições de P\&D;

9. projetos que visem o desenvolvimento de equipamentos fora de série para emprego em escala piloto, por instituições de P\&D. A meta correspondente a este objetivo é apoiar até 25 projetos.

\section{Quinto objetivo}

Fortalecer os serviços de apoio e suporte às atividades de pesquisa e desenvolvimento, alocando recursos para apoiar serviços de documentação e informação e à implementação e melhoria de Centrais Analíticas com o objetivo de atender à demanda nacional.

\section{Estratégia}

Em relação ao objetivo de fortalecimento de serviços de apoio e 
suporte, uma estratégia fundamental consiste em estabelecer facilidades instrumentais, em técnicas estabelecidas e de ampla utilização, necessárias para o trabalho de pesquisa e desenvolvimento em Química, bem como garantir o acesso de pesquisadores a fontes relevantes de informação primária (revistas, livros, monografias), a publicações de referência (Chemical Abstracts, Current Contents) e a bases de dados informatizadas.

Neste sentido, serão utilizados os seguintes meios:

- estabelecimento de organizações ou entidades prestadoras de serviços analíticos, tendo em vista levantamentos da demanda de serviços analíticos, em nível nacional ou regional, não atendida pelas facilidades e laboratórios existentes, levantamentos da oferta e da capacidade ociosa de serviços analíticos, por parte de laboratórios de IES, Institutos de Pesquisa e Empresas; - fortalecimento de bibliotecas, no que se refere ao seu acervo de obras especializadas de Química e Engenharia Química, bem como de infraestrutura de serviços especializados para a recuperação automatizada da informação.

\section{Metas (5 anos para comprometimento dos recursos)}

- apoiar até cinco Centrais analíticas regionais;

- fortalecer uma Biblioteca Principal de Química e Engenha-

ria Química;

- fortalecer até dez bibliotecas regionais;

- apoiar 15 outras bibliotecas.

\section{CONCLUSÃO}

Este planejamento pode não ter sido rigorosamente seguido durante todo o PADCT-II, porque ajustes tiveram que ser feitos durante sua execução. Serviu entretanto de um norte que orientou de perto sua implementação até o final. Os resultados do Subprograma QEQ no apoio a pesquisa acadêmica e formação de recursos humanos não podem ser questionados.

O Subprograma QEQ sempre foi um programa líder, afirmou o secretário executivo do PADCT, em artigo publicado em Química Nova ${ }^{8}$. Por isto mesmo, a comunidade perdeu muito com a falta de um PADCT-III, que nunca foi implementado. A escola que o PADCT representou deu muitos frutos. Grupos de pesquisa, consolidados ou em consolidação, continuam sendo apoiados, com outra denominação. A ênfase dada às bibliotecas certamente contribuiu para a criação do Portal de Periódicos da Capes. O apoio aos cursos de graduação talvez tenha contribuído para o importante trabalho que a SBQ tem feito no sentido de atualizar o conteúdo destes cursos. Porém, deixou saudade a participação ativa da comunidade na definição das prioridades.
Por outro lado, desde 1988, quando foi concluída a elaboração do Documento Básico, que o déficit na balança comercial da indústria química continua crescendo. Porém esta é uma visão pessimista. Conforme o Documento Básico, em 1988 as exportações de produtos químicos corresponderam 1,3 US\$ bilhões enquanto as importações custaram 2,6 US\$ bilhões. Já em 2006, conforme dados da ABIQUIM ${ }^{9}$, estes valores correspondem a 8,9 e 17,3 US\$ bilhões, respectivamente. Muito embora o déficit venha crescendo a uma taxa de U\$ 500 mil por ano, tendo aumentado de 1,3 para 8,4 US\$ bilhões, o importante é observar que a relação entre o custo das importações e o resultado das exportações, igual a 2, continua praticamente inalterado. É bem verdade que no intervalo entre estes dois extremos esta relação tenha crescido e chegado a $3 \mathrm{em}$ 2001, mas vem decrescendo regularmente desde então.

Se foi o déficit na balança comercial que levou o governo brasileiro a descobrir a importância da Química, é preciso que continue alerta para o fato de que o problema continua. Seria ingenuidade pensar que o apoio durante uns poucos anos pudesse alterar uma situação tão crítica. A propósito, seria interessante observar o que ficou registrado no Documento "Opportunities in Chemistry"10, elaborado em 1985 por um Comitê de especialistas do "National Research Council" norteamericano: "The U.S. chemical industry has a current $\$ 12$ billion positive balance of trade. Continued competitiveness depends upon constant improvement of existing processes and introduction of new ones. Advances in chemical catalysis and synthesis will be key to maintaining our current position of world leadership". Como é natural, cada país procura defender seus interesses, o apoio à Química por parte do governo precisa ser mantido, sob pena de vermos voltar a crescer esta desfavorável relação entre importações e exportações de produtos químicos.

\section{REFERÊNCIAS}

1. Alves, O. L.; Quim. Nova 2005, 28 (Supl.), S44

2. Gama, A. A. S.; Quim. Nova 2001, 24, 717

3. Galembeck, F. , Quim. Nova 2005, 28 (Supl.), S52.

4. Castro, L. A. B. ; Cybermetrics (Madrid) 2005, 9, 1.

5. Toma, H. E.; Ferreira, A. M. C.; Quim. Nova 2002, (25 Supl. 1), 66.

6. Schmal, M.; "História da Catálise no Brasil", disponível em http:// www.sbcat.org/sbcat/historiaacademia.php, acessada em Março 2007.

7. Lins, M. B.; Trópico e atuais orientações para pesquisa no Brasil. Em Seminário de Tropicologia: Questões da atualidade brasileira, 1982, Fundaj: Massangana: Recife, 1988, p. 211-225.

8. Castro, L. A. B.; Prescott, E.; Quim. Nova 1997, 20 (Especial),15.

9. http://www.abiquim.org.br/conteudo.asp?princ=ain\&pag=balcom, acessada em Março 2007

10. "Opportunities in Chemistry", Pimentel G. C. (Chairman) Committee to Survey Opportunities in the Chemical Sciences, National Academic Press: Washington, D. C, 1985, p. 6. 